\title{
Magnon Landau levels in the strained antiferromagnetic honeycomb nanoribbons
}

\author{
Junsong Sun \\ School of Physics, Beihang University, Beijing 100191, China \\ Huaiming Guo* \\ School of Physics, Beihang University, Beijing 100191, China \\ and Beijing Computational Science Research Center, Beijing 100193, China \\ Shiping Feng $\mathbb{B}$ \\ Department of Physics, Beijing Normal University, Beijing 100875, China
}

(Received 23 September 2021; accepted 14 December 2021; published 27 December 2021)

\begin{abstract}
The pseudomagnetic field created by a nonuniform uniaxial strain is introduced into the antiferromagnetic honeycomb nanoribbons. The formation of magnon pseudo-Landau levels, which appear from the upper end of the spectrum and whose level spacings are proportional to the square root of the level index, is revealed by the linear spin-wave theory. The antiferromagnetic order is gradually weakened along the $y$-direction by the strain. At large enough strength, the system is decoupled into isolated zigzag chains near the upper boundary and demonstrates one-dimensional magnetic property there. While the quantum Monte Carlo simulations also predict such a transition, this exact method gives a critical point deeper in the bulk. We also investigate the $X Y$ antiferromagnetic honeycomb nanoribbons and find similar pseudo-Landau levels and antiferromagnetic evolution. Our results unveil the effect of a nonuniform uniaxial strain on the spin excitations and may be realized experimentally based on two-dimensional quantum magnetic materials.
\end{abstract}

DOI: 10.1103/PhysRevResearch.3.043223

\section{INTRODUCTION}

Mechanical strain has become a powerful tool to engineer the electronic property of graphene and other two-dimensional (2D) quantum materials [1-4]. The low-energy physics of graphene is described by the Dirac Hamiltonian, in which the perturbation of a strain acts as a vector potential with opposite signs at the two valleys [5,6]. Experimentally, a controlled uniaxial strain can be readily realized in graphene using feasible techniques. However, such a strain results in a constant gauge field, which shift the position of the Dirac points in opposite directions and can induce a band gap only at unrealistic large strength [7,8].

A nonzero pseudomagnetic field (PMF), especially a uniform one which can mimic the effect of a real magnetic field, is highly desirable. Since the PMF magnitude is proportional to the gradient of the strain, a PMF should be created by a nonuniform strain $[9,10]$. Guinea et al. first predicted a triaxial strain can lead to a strong uniform PMF [2]. Later, experimentally more available approaches, such as bending or twisting graphene [11-14], were proposed to generate an almost uni-

\footnotetext{
*hmguo@buaa.edu.cn

Published by the American Physical Society under the terms of the Creative Commons Attribution 4.0 International license. Further distribution of this work must maintain attribution to the author $(s)$ and the published article's title, journal citation, and DOI.
}

form PMF. While it is still challenging to directly realize the above theoretical proposals, the pseudo-Landau levels (PLLs) induced by PMFs have been observed by scanning tunneling microscopy in highly localized regions of graphene with nonplanar deformations [15-17].

Recently strain-induced gauge fields have been generalized to three-dimensional (3D) Dirac and Weyl semimetals [18-20], and even neutral quasiparticles such as Bogoliubov particles in 2D nodal superconductors [21] and magnons in honeycomb antiferromagnets [22-26]. Although Landau quantization of neutral quasiparticles is formed by the straininduced PMF, its properties are not completely identical to those in graphene. Specifically, under PMF induced by the triaxial strain in a honeycomb antiferromagnet, the PLLs appear at the upper end of the magnon spectrum and are equally spaced [27,28]. The strain is introduced to the honeycomb antiferromagnet by analogizing the exchange coupling like the hopping amplitude in graphene and modifying it in the same way as the latter. The strain-induced gauge field may not act on the magnons exactly as it does in graphene. Hence it is natural to ask whether the different methods used to engineer a uniform PMF in graphene have the same effect on the spin excitations in honeycomb antiferromagnets.

In this paper, we study the Heisenberg and $X Y$ Hamiltonians on the honeycomb lattice under a nonuniform uniaxial strain utilizing the linear spin-wave theory (LSWT) and quantum Monte Carlo (QMC) method. First, the formation of PLLs is revealed using LSWT. Then we address the evolution of the antiferromagnetic (AF) order, which is characterized by 
a finite local magnetization and long-range AF correlations. Both approaches show the AF order is reduced monotonically in the $y$-direction and predict a critical position beyond which the system is described by decoupled one-dimensional (1D) Heisenberg chains for large enough strain strength. Finally, we present the results of the $X Y$ Hamiltonian under the same kind of strain. Here the formation of PLLs and the evolution of the AF order with the strain are very similar to the Heisenberg case, except that the magnetic order is more robust and persists even at the largest possible strain strength. These results are closely related to the $2 \mathrm{D}$ quantum magnetic materials and will attract both theoretical and experimental interests.

This paper is organized as follows. Section II introduces the precise model we will investigate, along with our computational methodology. Section III presents the magnon Landau levels in LSWT. Section IV uses LSWT and QMC simulations to study the evolution of the AF order. Section $\mathrm{V}$ demonstrates the results of the $X Y$ Hamiltonian under a uniaxial strain. Section VI contains further discussion and conclusions.

\section{THE MODEL AND METHOD}

We consider an AF Heisenberg model on a honeycomb nanoribbon, which in the absence of strain is written as

$$
H_{0}=J \sum_{\langle i j\rangle} \mathbf{S}_{i} \cdot \mathbf{S}_{j},
$$

where $J$ is the AF exchange coupling; $\mathbf{S}_{i}=\left(S_{i}^{x}, S_{i}^{y}, S_{i}^{z}\right)$ is the spin- $\frac{1}{2}$ operator on the site $i$, which obeys commutation relations, $\left[S_{i}^{\mu}, S_{j}^{\nu}\right]=i \hbar \varepsilon_{\mu \nu \tau} S_{i}^{\tau} \delta_{i j}$ with $\varepsilon_{\mu \nu \tau}$ the Levi-Civita symbol, and $\mu, v, \tau=x, y, z$ representing spin components. In the presence of strain, the lattice is deformed and the Hamiltonian is modified through a simple modulation of the exchange couplings. For small displacements, we have

$$
J \longrightarrow J_{i j}=J\left(1-\gamma \Delta u_{n}\right),
$$

where $\gamma$ represents the strength of magnetoelastic coupling; $\Delta u_{n}(n=1,2,3)$ is the relative displacement of the bond, given by

$$
\Delta u_{n}=\sum_{i, j} \frac{a_{n}^{i} a_{n}^{j}}{a_{0}^{2}} \epsilon_{i j} .
$$

In the above equation, $\vec{a}_{n}$ are the nearest-neighbor vectors, and the strain tensor is $\epsilon_{i j}=\frac{1}{2}\left[\partial_{j} u_{i}+\partial_{i} u_{j}\right](i, j=x, y)$ with the displacement $\vec{u}(\mathbf{r})=\left[u_{x}(\mathbf{r}), u_{y}(\mathbf{r})\right]$ of the lattice site at position $\mathbf{r}=(x, y)$. Here $\vec{u}(\mathbf{r})$ is assumed to depend only on $y$, which results in $\epsilon_{x x}=\epsilon_{x y}=\epsilon_{y x}=0$ and $\Delta u_{1}=\epsilon_{y y}, \Delta u_{2}=$ $\Delta u_{3}=\epsilon_{y y} / 4$ [29]. The strain tensor is expected to generate a pseudogauge field

$$
\mathbf{A}=\frac{\gamma}{2}\left(\begin{array}{c}
\epsilon_{x x}-\epsilon_{y y} \\
-2 \epsilon_{x y}
\end{array}\right)
$$

For our case, only $A_{x}=-\frac{\gamma}{2} \epsilon_{y y}$ is nonzero. We take $\epsilon_{y y}=$ $\frac{c}{\gamma} y$, which generates a homogeneous pseudomagnetic field $\vec{B}=\frac{1}{2} c \hat{z}$. We set the bottom of the ribbon as the coordinate origin of the $y$-direction, thus $y_{j}=\frac{3}{2}(j-1)+\frac{1}{2}$ for the blue atoms in the $j$ th zigzag horizontal chain (see Fig. 1). Since the exchange coupling of the vertical bond decreases more

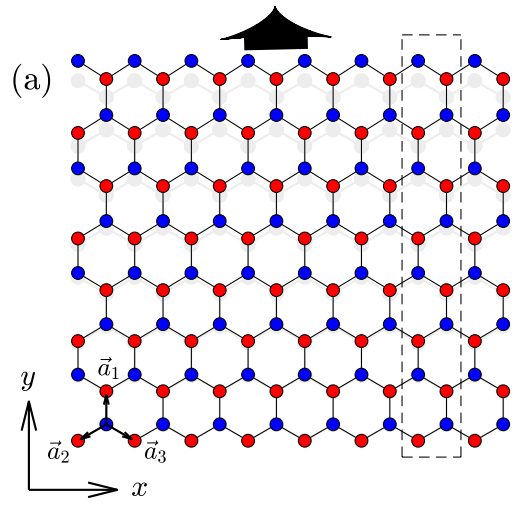

(b)

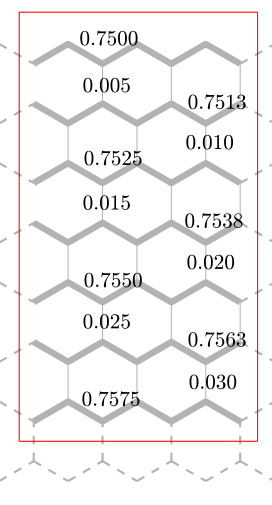

FIG. 1. (a) Schematic representation of a strained honeycomb nanoribbon with zigzag boundaries. The nanoribbon is periodic along the $x$-direction, and the width in the $y$-direction is $L_{y}=8$. (b) Enlarged plot in the vicinity of the upper boundaries of a $L_{y}=$ 200 honeycomb nanaribbon. The value of the exchange coupling on each bond is represented by the thickness of the bond and marked explicitly near the bonds. $c / c_{\max }=1$ is used for the strain strength in (b).

rapidly with $y$, the maximum strain parameter $c_{\max }$ is determined by the appearance of zero exchange coupling on the bonds along the $y$-direction. For a ribbon with fixed width $L_{y}$, the vertical bonds connecting the blue sites at the upper boundary are expected to vanish at the maximum strain strength, i.e., $J\left(1-\gamma \Delta u_{1}\right)=J\left(1-c_{\max } y_{\max }\right)=0$, where the maximum $y$-coordinate is $y_{\max }=\frac{3}{2}\left(L_{y}-1\right)+\frac{1}{2}$. Hence we have $c_{\max }=1 / y_{\max }$, which we take as the scale of the strain parameter $c$ throughout the paper.

In the following discussions, we study the model in Eq. (1) under the above nonuniform uniaxial strain using LSWT and stochastic series expansion (SSE) QMC method with directed loop updates [30,31]. The SSE method expands the partition function in power series, and the trace is written as a sum of diagonal matrix elements. The directed loop updates make the simulation very efficient [32-34]. Our simulations are on a honeycomb nanoribbon with the total number of sites $N_{s}=L_{x} \times L_{y}$ with $L_{x}=20, L_{y}=200$ the linear sizes. The nanoribbon is periodic (open) along the $x(y)$ direction. There are no approximations causing systematic errors, and the discrete configuration space can be sampled without floating point operations. The temperature is set to be $\beta=200$, which is low enough to obtain the ground-state properties.

\section{MAGNON LANDAU LEVELS IN THE LINEAR SPIN WAVE THEORY}

Let us first investigate the physical properties of the strained model (1) using LSWT, where the spin operators are replaced by bosonic ones via Holstein-Primakoff (HP) transformation [35]. The transformation on sublattice A (the spin is in the positive $z$-direction) is defined as

$$
\begin{aligned}
S_{i}^{+} & =\sqrt{2 S} a_{i}, S_{i}^{-}=\sqrt{2 S} a_{i}^{\dagger}, \\
S_{i}^{z} & =S-a_{i}^{\dagger} a_{i} .
\end{aligned}
$$



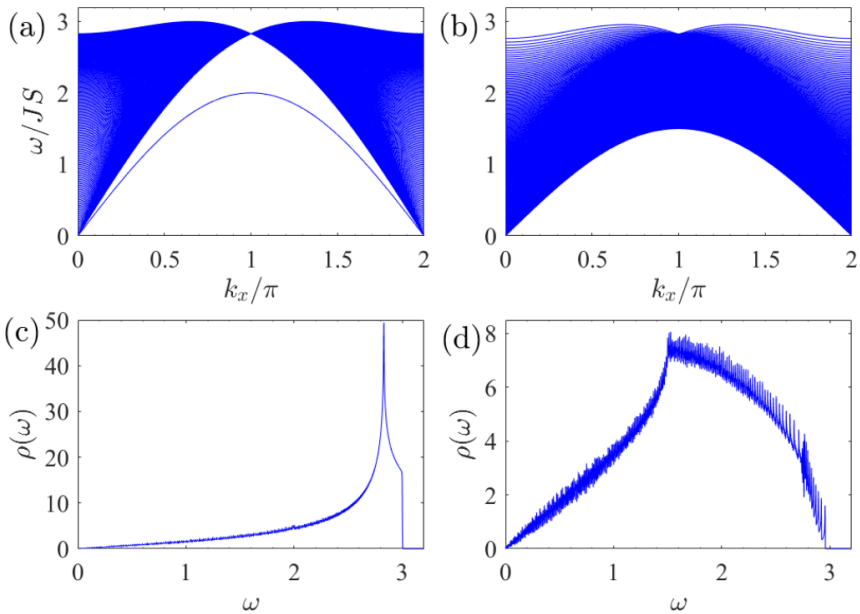

FIG. 2. The magnon spectrum of the AF honeycomb nanoribbon: (a) without the strain and (b) in the presence of a nonuniform uniaxial strain. Panels (c) and (d) are the corresponding magnon density of states of (a) and (b), respectively. The strain strength in (b) and (d) is $c / c_{\max }=1$.

On sublattice B (the spin is in the negative $z$-direction), the spin operators are defined as

$$
\begin{aligned}
S_{i}^{+} & =\sqrt{2 S} b_{i}^{\dagger}, S_{i}^{-}=\sqrt{2 S} b_{i}, \\
S_{i}^{z} & =b_{i}^{\dagger} b_{i}-S .
\end{aligned}
$$

Keeping only the bilinear terms, the bosonic tight binding Hamiltonian reads

$$
H=\sum_{\langle i j\rangle} J_{i j} S\left(a_{i} b_{j}+a_{i}^{\dagger} b_{j}^{\dagger}+a_{i}^{\dagger} a_{i}+b_{j}^{\dagger} b_{j}\right) .
$$

Performing a Fourier transformation in the $x$-direction and under the basis $X_{k_{x}}^{\dagger}=\left(a_{1, k_{x}}^{\dagger}, b_{1, k_{x}}, \ldots, a_{L_{y}, k_{x}}^{\dagger}, b_{L_{y}, k_{x}}\right)$, the above Hamiltonian is written as $H=\sum_{k_{x}} X_{k_{x}}^{\dagger} M\left(k_{x}\right) X_{k_{x}}$, where $M\left(k_{x}\right)$ is a $2 L_{y} \times 2 L_{y}$ matrix. By a standard Bogliubov transformation [36,37], the matrix $M\left(k_{x}\right)$ becomes diagonal, and the magnon spectra are directly obtained.

The open boundaries are created by breaking the bonds connecting the outmost sites of the zigzag edges. As shown in Fig. 2(a), a new branch of modes associated with the boundaries appear below the bulk spectrum [38]. Their boundary nature is further revealed by the distribution of the corresponding wave functions, which is mainly localized near the boundaries. The density of states (DOS) is plotted in Fig. 2(c), which resembles that of itinerant electrons in graphene. As expected, the low-energy linear behavior in DOS is due to the linear dispersion of the magnon excitation in the antiferromagnets. Also, the saddle point at $k_{x}=0$ leads to a Van Hove singularity in the magnon spectrum [39].

After the strain is applied, the degeneracy of the energy levels is removed, and the spectrum becomes much broader [see Fig. 2(b)]. This change is most evident at $k_{x}=\pi$, where all energy levels are originally degenerate in the absence of strain (see Appendix A). In particular, the magnon spectrum is flatted by the strain, and DOS exhibits oscillating behavior. The appearance of sharp peaks in the magnon DOS should result from the flat levels, thus this is direct evidence of the
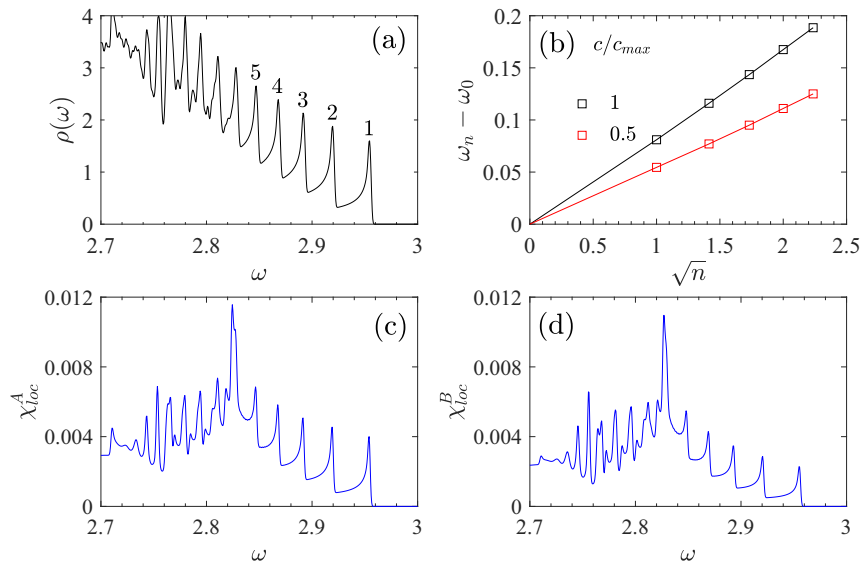

FIG. 3. (a) The density of states near the upper end of the magnon spectrum. (b) The PLL energy $\omega_{n}$ as a function of the square root of the level index $n$. The solid lines represent linear fitting of the data. Local susceptibility at the center of the unit cell on the (c) A sublattice and (d) B sublattice. The linear size is $L_{x}=20, L_{y}=200$. In (a), (c), and (d), the strain strength is $c / c_{\max }=1$.

formation of the magnon PLLs. As shown in Fig. 3(a), the magnon PLLs appear from the upper end of the spectrum, which is in agreement with the recent studies on the Heisenberg model under a triaxial strain. However, by fitting the positions of the peaks, it is found that the PLL energy $\omega_{n}$ is proportional to the square root of the level index $n$, which is in great contrast to the equally spaced PLLs in honeycomb antiferromagnets under a triaxial strain. In addition, the scope increases when enhancing the applied strain. Except for the apparent position, these properties are very similar to the Landau levels of Dirac fermions in graphene [40] (an analytical understanding is presented in Appendix B).

The appearance of the magnon PLLs can also be reflected in the local susceptibility, which is defined as [41]

$$
\chi_{\mathrm{loc}}^{i}(\omega)=\int_{-\infty}^{\infty} d t e^{i \omega t}\left\langle S_{i}^{x}(t) S_{i}^{x}+S_{i}^{y}(t) S_{i}^{y}\right\rangle .
$$

In LSWT, the local susceptibility is formulated in terms of the $\delta$ functions peaked at the magnon eigenvalues, and hence is equivalent to the density of states in characterizing the flat PLLs. More importantly, the local susceptibility can be exactly determined by numerical analytic continuation of the imaginary time spin correlations obtained by the QMC simulation. The local susceptibilities on $A$ - and $B$-sublattice sites deep in the lattice are shown in Figs. 3(c) and 3(d). Indeed, both of them demonstrate sharp peaks at exactly the same positions as those in DOS, further confirming the formation of the magnon PLLs.

\section{THE EVOLUTION OF THE AF ORDER}

We next study how the AF order is affected by the strain. In LSWT, the existence of Néel order is identified by a finite local magnetization. Since the honeycomb nanoribbon is translation invariant in the $x$-direction, the local magnetization varies only within the unit cell which extends over the entire width of the ribbon (see Fig. 1). Figure 4 shows the local magnetization as a function of site index in the unit cell at 

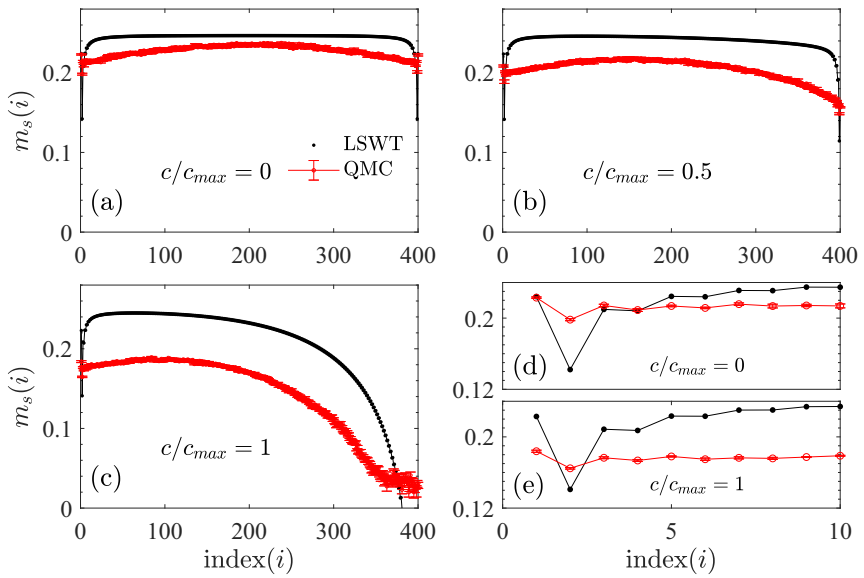

FIG. 4. The distribution of the local magnetization obtained by LSWT and QMC simulations at the strain strength (a) $c / c_{\max }=0$, (b) 0.5 , and (c) 1. Panels (d) and (e) enlarge the curves near the lower boundary in (a) and (c), respectively. Here the index range is up to $2 L_{y}=400$ (the same with the figures hereafter), which is due to the existence of the sublattice degree.

several values of the strain strength. In the absence of strain, the Néel orders near the boundaries are perturbed, and the values gradually decrease as the sites approach the boundaries. Nevertheless, $m_{s}(i)$ is always finite and becomes almost uniform away from the boundaries, implying the long-range $\mathrm{AF}$ order still is preserved in the presence of open boundaries. It is noted that the local magnetization on the outmost sites of the boundary is much larger than that of its nearby sites [see Figs. 4(d) and 4(e)]. The two outmost sites represent the two sublattices of the boundary zigzag chain. Although it is antiferromagnetic along the 1D chain, the magnetic moments are unequal within the two-site unit cell, resulting in a net ferromagnetic moment. Hence a ferrimagnetic order is formed along the zigzag boundary, which has also been revealed in the Hubbard model on honeycomb nanoribbons [42-46].

After the strain is applied, the value of the local magnetization monotonically decreases with the strain strength. Since the exchange coupling is gradually reduced in the $y$-direction, the magnetization is more affected on the sites father away from the lower boundary. In particular, at large enough strain strength and near the upper boundary the local magnetization decreases rapidly and becomes negative at a critical position, implying the $\mathrm{AF}$ order vanishes hereafter. This behavior is due to the exchange couplings of the vertical bonds becoming negligibly small near the upper boundary, and the system can be regarded as a collection of isolated 1D Heisenberg chains, resulting in the breakdown of $2 \mathrm{D} \mathrm{AF}$ order there. In contrast, the region near the lower boundary is less affected since the exchange couplings here are least modified. While the LSWT can qualitatively demonstrate the evolution of the AF order with the strain, the exact results should be obtained by the unbiased QMC simulations.

In QMC simulations, the local value of the magnetization is given by $m_{s}^{\mathrm{qmc}}(i)$, defined as [47]

$$
m_{s}^{\mathrm{qmc}}(i)=\sqrt{\frac{3}{N} \sum_{j=1}^{N} \operatorname{sgn}(i, j)\left\langle S_{i}^{z} S_{j}^{z}\right\rangle},
$$
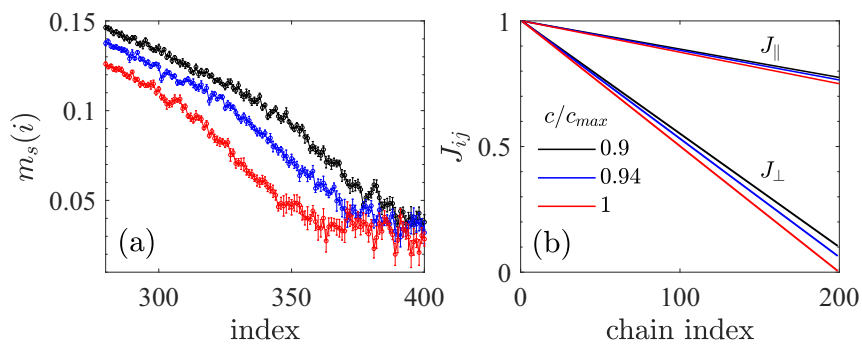

FIG. 5. (a) The local magnetization obtained by QMC near the critical position for various strain strengths. (b) The parallel and transverse exchange couplings as a function of the zigzag chain index.

where the sum is over all lattice sites $j$, and $\operatorname{sgn}(i, j)=1(-1)$ if $i, j$ belong to the same (opposite) sublattice. Figure 4 plots the values of $m_{s}^{\mathrm{qmc}}(i)$ at the same strain strengths as those in LSWT. The QMC values are smaller than the LSWT ones. Also, the difference between the values from the two approaches increases as the strain is strengthened. In the absence of strain, the QMC curve slowly increases and gets a maximum at the central point. In contrast, the LSWT one is nearly flat in most of the bulk region. Here it is noted that the QMC and LSWT results are only slightly different, and most of the values from the two approaches have less than a $10 \%$ difference. This implies the linear approximation in the HP transformation is pretty accurate, which has also been found in the existing literature $[48,49]$. For the strain strength $c / c_{\max }=1$, a clear transition is visible in the QMC curve near the upper boundary.

Figure 5(a) plots the local magnetization obtained by QMC near the critical position for various strain strengths. It shows the crossover from 2D to 1D behavior is continuous, which may be due to that the exchange coupling varies smoothly all the way down to a very small value with the coordinate $y$ [see Fig. 5(b)]. We cannot determine the exact critical strain after which there appears such a transition. Nevertheless, since the transition has already become indistinguishable at $c / c_{\max }=0.9$, the critical value should be pretty large. While such a transition is also predicted by LSWT with $m_{s}(i)=0$, the QMC transition happens a bit deeper in the ribbon than the LSWT one. These results imply that although the quantum fluctuation is omitted, LSWT can still give a qualitatively correct evolution of the AF order.

How the magnetic property is affected by the strain can also be demonstrated by the spin correlation $C(i, j)=\left\langle S_{i}^{z} S_{j}^{z}\right\rangle$. Figure 6 plots the spin correlation between two sites within the super unit cell at $c / c_{\max }=1$ for the Heisenberg Hamiltonian. When the reference point $i_{0}$ is in the middle of the unit cell, $C\left(i_{0}, j\right)$ is always finite for $j<i_{0}(j$ is located in the lower part), but it gradually decreases in the upper part and becomes nearly zero from a critical position. In contrast, for a reference point $i_{1}$ near the upper boundary, $C\left(i_{1}, j\right)$ reduces to zero quickly as $j$ goes away from $i_{1}$. We also plot $C(i, j)$ with both $i, j$ on the same zigzag chain, which stays finite even for the largest distance. These results are consistent with the occurrence of a crossover from 2D to 1D magnetic properties at the critical position. Moreover, it is noted in Fig. 5(a) that the curve begins to decrease in a slower way after the 

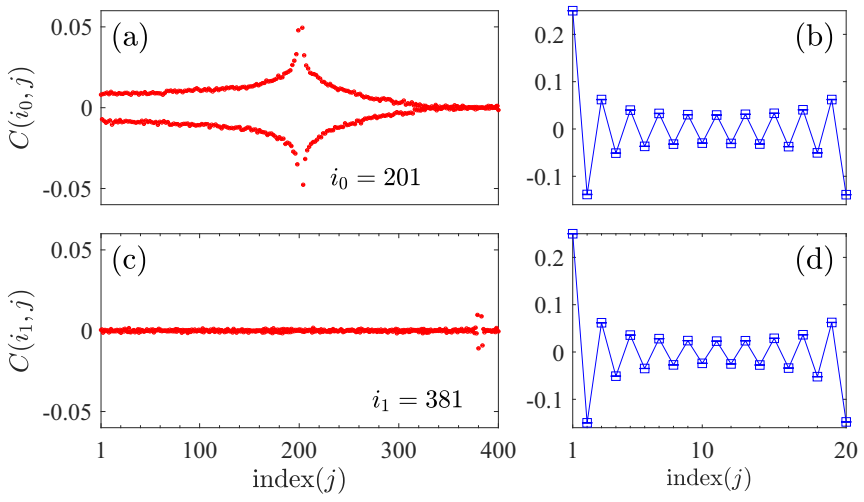

FIG. 6. The spin correlation with the reference point fixed at the middle point $i_{0}$ of the unit cell: (a) $j$ varies within the unit cell, (b) $j$ is on the same zigzag chain with $i_{0}$ (here $i_{0}$ corresponds to the $j=1$ site). The reference site is changed to a near-boundary site $i_{1}$ in (c) and (d), which are the corresponding plots of (a) and (b), respectively.

critical position. This can be understood in terms of the spin correlations. Since the vertical exchange coupling has become negligibly weak near the upper boundary, the spin correlation in this direction is nearly zero. In contrast, the spin correlations along the zigzag chain are still considerably large, which actually dominates the local magnetization. The parallel spin correlations vary slowly with $y$ here, and so does the local magnetization. Due to the contribution from the parallel spin correlations, the local magnetization still has a finite small value.

\section{THE STRAINED $X Y$ ANTIFERROMAGNETIC HONYCOMB NANORIBBON} by

We next consider the spin- $\frac{1}{2} X Y$ AF Hamiltonian described

$$
H_{X Y}=J \sum_{\langle i j\rangle}\left(S_{i}^{x} S_{j}^{x}+S_{i}^{y} S_{j}^{y}\right) .
$$

By a rotation of the coordinate system, the above model becomes the $X Z$ Hamiltonian [50]:

$$
\begin{aligned}
H_{X Z} & =J \sum_{\langle i j\rangle}\left(S_{i}^{x} S_{j}^{x}+S_{i}^{z} S_{j}^{z}\right), \\
& =J \sum_{\langle i j\rangle}\left(S_{i}^{z} S_{j}^{z}+\frac{1}{4} \sum_{\mu, \nu= \pm} S_{i}^{\mu} S_{j}^{\nu}\right) .
\end{aligned}
$$

Under a nonuniform uniaxial strain, the same modulation of the exchange coupling with that in Eq. (2) can be made, and the application of LSWT is straightforward.

Figure 7(a) plots the magnon density of states. Under PMF induced by the strain, sharp peaks appear from the upper end of the spectrum, marking the formation of PLLs. Also, the energies of PLLs are proportion to $\sqrt{n c}$ [ $n$ is the level index, and the PMF magnitude is proportional to the strain strength $c$; see Fig. 7(b)], which is very similar to the results from the strained Heisenberg model. However, such properties are in great contrast to the situation under a triaxial strain, where
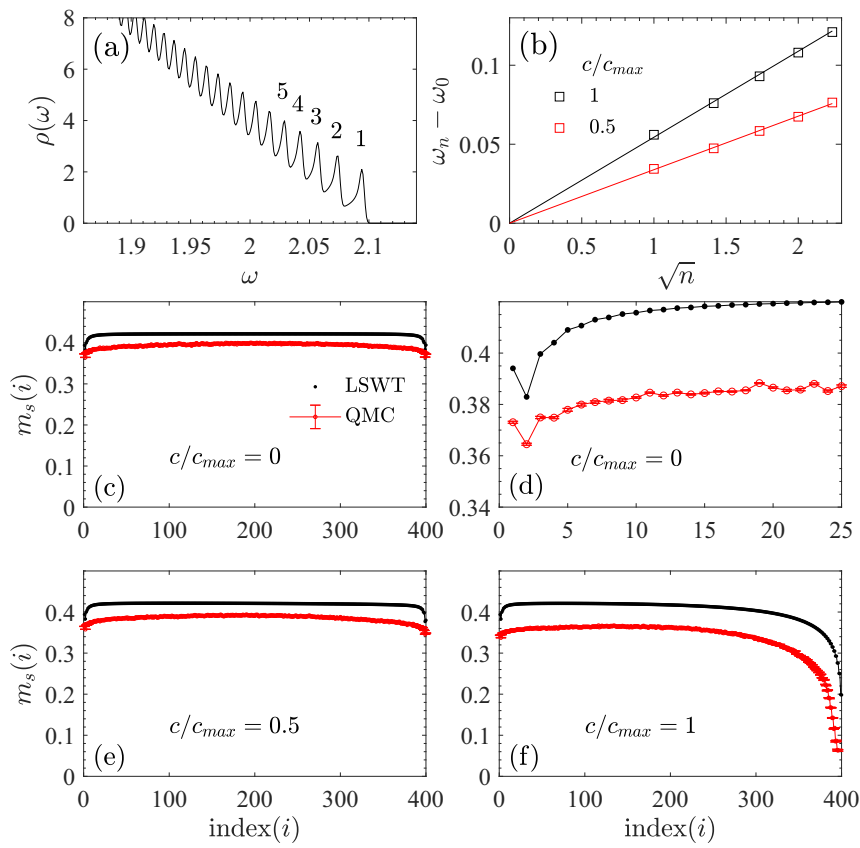

FIG. 7. (a) The magnon density of states near the upper end of the spectrum of the strained $X Y$ Hamiltonian. (b) The PLL energy $\omega_{n}$ as a function of the square root of the level index $n$. The distribution of the local magnetization obtained by LSWT and QMC simulations at the strain strength $c / c_{\max }$ : (c) 0 ; (e) 0.5 ; (f) 1 . Panel (d) enlarges the curves of (c) near the lower boundary. In (a), the strain strength is $c / c_{\max }=1$.

PLLs appear from the middle of the spectrum and the peaks follow the relations $\propto n^{\frac{1}{3}}, n^{\frac{2}{3}}$ [28].

We then investigate how the strain affects the AF order. Similarly, the local magnetization decreases monotonically in the $y$-direction near the upper boundary in the presence of strain. Compared to the Heisenberg case, the values of the local magnetization are much larger at the same condition. Also, as shown in Fig. 7(f), even at the largest strain strength when the $y$-direction bonds near the upper boundary are considerably weak, the local magnetization always stays finite, suggesting the long-range AF order is preserved in the whole system. This implies the $X Y$ Hamiltonian is more robust to the modulation of the exchange couplings induced by the strain [51]. Qualitatively, the reason is that there are three (two) spin components in the Heisenberg $(X Y)$ case, thus the quantum fluctuation is much stronger in the Heisenberg model than that in the $X Y$ one. The related quantity defined in Eq. (9) is also calculated using the QMC methods. While the QMC results are qualitatively consistent with those from LSWT, QMC gives relatively smaller values at the same strain strengths.

\section{CONCLUSIONS}

The magnon PLLs and the evolution of the AF order in the strained AF honeycomb nanoribbons are studied using LSWT and QMC simulations. After the strain is applied, the magnon PLLs are formed from the upper end of the spectrum, and their level spacings are proportional to the square root of the level index. Since the exchange couplings are linearly weakened by 
the uniaxial strain, the local magnetization decreases monotonically in the $y$-direction. Specifically, at large enough strain strength, the $y$-direction bonds near the upper boundary become negligibly weak such that the system there is decoupled into isolated zigzag chains, exhibiting 1D antiferromagnetic property. The $X Y$ Hamiltonian under the same kind of strain demonstrates similar properties except that the AF order is more robust than the Heisenberg case. The behavior of the $X Y$ case is in great contrast to that under a triaxial strain, where the PLLs appear from the middle of the spectrum and the peaks follow a third-root relation.

In the past several years, significant progress has been achieved in the field of $2 \mathrm{D}$ quantum magnetic materials [52-55]. 2D magnetic order has been observed in various magnetic van der Waals materials, and most of them form with the magnetic elements in a honeycomb lattice [56-60]. All the 2D magnetism can, in principle, be described by three fundamental models: Ising, $X Y$, or Heisenberg. Strain engineering, as an important approach to control and manipulate magnetic states, has been widely adopted in the research of 2D magnetic materials [61-64]. Thus the new physical phenomena induced by the engineered strain would definitely be interesting to the related experiments. Moreover, the present study will contribute to the theoretical understanding of the behavior of the neutral quasiparticles in pseudomagnetic fields, and proposes an alternative routine to manipulate magnons, which may have potential applications in designing new devices in magnon spintronics $[65,66]$.

\section{ACKNOWLEDGMENTS}

The authors thank Tianyu Liu, Yancheng Wang, Wen Yang, Chenyue Wen, and Xingchuan Zhu for helpful discussions. J.S. and H.G. acknowledge support from the National Science Foundation of China (NSFC) Grants No. 11774019 and 12074022, the NSAF, part of the NSFC, Grant No. U1930402, the Fundamental Research Funds for the Central Universities and the HPC resources at Beihang University. S.F. is supported by the National Key Research and Development Program of China and the NSFC under Grants No. 11974051 and 11734002 .

\section{APPENDIX A: THE ANALYTICAL SOLUTION OF THE MAGNON EIGENVALUES AT $k_{x}=\pi$ in Fig. 2(a)}

In the absence of strain, the Hamiltonian matrix of Eq. (7) in the momentum space reads as

$$
M\left(k_{x}\right)=J S\left(\begin{array}{ccccccc}
2 & \gamma_{k_{x}} & 0 & 0 & 0 & 0 & \ldots \\
\gamma_{k_{x}}^{*} & 3 & 1 & 0 & 0 & 0 & 0 \\
0 & 1 & 3 & \gamma_{k x}^{*} & 0 & 0 & 0 \\
0 & 0 & \gamma_{k x} & 3 & 1 & 0 & 0 \\
0 & 0 & 0 & 1 & 3 & \gamma_{k x} & 0 \\
0 & 0 & 0 & 0 & \gamma_{k x}^{*} & 3 & \ldots \\
\vdots & 0 & 0 & 0 & 0 & \vdots & \ddots
\end{array}\right),
$$

where $\gamma_{k_{x}}=1+e^{-i k_{x}}$. At $k_{x}=\pi$, we have $\gamma_{k_{x}=\pi}=0$, thus $M\left(k_{x}\right)$ is a block diagonal. There are two eigenvalues: $2 J S, 2 \sqrt{2} J S$, between which the value $2 \sqrt{2} J S$ has a large degeneracy. The strain breaks the degeneracy, and the spectrum is broadened at $k_{x}=\pi$, as shown in Fig. 2(b).

\section{APPENDIX B: THE ANALYTICAL TREATMENT BASED ON THE EFFECTIVE HAMILTONIAN NEAR THE DIRAC POINT}

Here we present the analytical treatment of magnon pseudo-Landau levels in the stained quantum antiferromagnetic Heisenberg model based on the effective Hamiltonian near the Dirac point.

\section{The low-energy effective Hamiltonian near the Dirac point}

After the spin operators are replaced by bosonic ones via Holstein-Primakoff transformation, we obtain the following bosonic tight-binding Hamiltonian:

$$
\begin{aligned}
H= & J_{1} S \sum_{i, j}\left(a_{i}^{\dagger} b_{j}^{\dagger}+a_{i} b_{j}+a_{i}^{\dagger} a_{i}+b_{j}^{\dagger} b_{j}\right) \\
& +J_{2} S \sum_{i, j}\left(a_{i}^{\dagger} b_{j}^{\dagger}+a_{i} b_{j}+a_{i}^{\dagger} a_{i}+b_{j}^{\dagger} b_{j}\right) \\
& +J_{3} S \sum_{i, j}\left(a_{i}^{\dagger} b_{j}^{\dagger}+a_{i} b_{j}+a_{i}^{\dagger} a_{i}+b_{j}^{\dagger} b_{j}\right) .
\end{aligned}
$$

In the momentum space, the Hamiltonian becomes $H=$ $\sum_{\mathbf{k}} \hat{\Psi}_{\mathbf{k}}^{\dagger} h(\mathbf{k}) \hat{\Psi}_{\mathbf{k}}$ with the basis $\hat{\Psi}_{\mathbf{k}}^{\dagger}=\left(a_{\mathbf{k}}^{\dagger} b_{\mathbf{k}}\right)$ and

$$
\begin{aligned}
h(\boldsymbol{k}) & =d_{x}(k) \sigma_{x}+d_{y}(k) \sigma_{y}+\left(J_{1}+2 J_{2}\right) S \sigma_{0}, \\
d_{x}(\boldsymbol{k}) & =J_{1} S \cos k_{y}+2 J_{2} S \cos \frac{\sqrt{3} k_{x}}{2} \cos \frac{k_{y}}{2}, \\
d_{y}(\boldsymbol{k}) & =-J_{1} S \sin k_{y}+2 J_{2} S \cos \frac{\sqrt{3} k_{x}}{2} \sin \frac{k_{y}}{2},
\end{aligned}
$$

where $\sigma_{x, y}$ are the Pauli matrices, $\sigma_{0}$ is the identity matrix, and $J_{n}=J\left(1-\gamma \Delta u_{n}\right)$ with $\Delta u_{1}=\epsilon_{y y}, \Delta u_{2}=\Delta u_{3}=\epsilon_{y y} / 4$. Writing the momentum near the Dirac point $\boldsymbol{K}=\left(\frac{4 \pi}{3 \sqrt{3}}, 0\right)$ as $\boldsymbol{k}=\boldsymbol{K}+\boldsymbol{q}$, and expanding $d_{y}(\boldsymbol{k}), d_{x}(\boldsymbol{k})$ to linear order of $\mathbf{q}$, the resulting Hamiltonian is

$$
\begin{aligned}
h(\boldsymbol{q})= & -\frac{3}{2} J S\left[\left(1-\frac{1}{4} \epsilon_{y y}\right) q_{x}+\frac{1}{2} \epsilon_{y y}\right] \sigma_{x} \\
& -\frac{3}{2} J S\left[\left(1-\frac{3}{4} \epsilon_{y y}\right) q_{y}+\frac{q_{x} q_{y}}{2}\left(1-\frac{1}{4} \epsilon_{y y}\right)\right] \sigma_{y} \\
& +J S\left(3-\frac{3}{2} \epsilon_{y y}\right) \sigma_{0} .
\end{aligned}
$$

The strain tensor is expected to generate a pseudomagnetic field, and the vector potential is

$$
\vec{A}=\frac{\gamma}{2}\left(\begin{array}{c}
\epsilon_{x x}-\epsilon_{y y} \\
-2 \epsilon_{x y}
\end{array}\right) .
$$

We choose $\epsilon_{y y}=\frac{c}{\gamma} y$ to get a homogeneous field $\vec{B}=\frac{1}{2} c \hat{z}$. Introducing $p=\frac{1}{2}-\frac{q_{x}}{4}, s=1+\frac{q_{x}}{2}, r=\frac{3}{4}+\frac{q_{x}}{8}$, and changing $y \rightarrow y+\frac{s}{r c}, k_{y} \rightarrow-i \partial_{y}$, we get the effective Hamiltonian near 
the Dirac point,

$$
\begin{aligned}
h\left(q_{x}\right)= & -\frac{3}{2} J S\left\{\left[q_{x}+p c\left(y+\frac{s}{r c}\right)\right] \sigma_{x}+i r c y \partial_{y} \sigma_{y}\right\} \\
& +3 J S\left(1-\frac{1}{2} c y\right) \sigma_{0} .
\end{aligned}
$$

\section{A solvable case without the $y$-dependent term before $\sigma_{0}$}

We first consider a specific solvable case: the $y$-dependent term before $\sigma_{0}$ is dropped artificially. The Hamiltonian reads as

$$
h\left(q_{x}\right)=-\frac{3}{2} J S\left\{\left[q_{x}+p c\left(y+\frac{s}{r c}\right)\right] \sigma_{x}+i r c y \partial_{y} \sigma_{y}\right\}+3 J S \sigma_{0} .
$$

We consider the eigenvalue problem

$$
\tau_{z} h\left(q_{x}\right)\left(\begin{array}{l}
\phi_{A}(y) \\
\phi_{B}(y)
\end{array}\right)=E\left(\begin{array}{l}
\phi_{A}(y) \\
\phi_{B}(y)
\end{array}\right),
$$

where $\tau_{z}$ is the Pauli matrix. Expanding the above matrixvector multiplication, two first-order differential equations are obtained,

$$
\begin{gathered}
-\frac{3}{2}\left[q_{x}+p c\left(y+\frac{s}{r c}\right)+r c\left(y \partial_{y}+\frac{1}{2}\right)\right] \phi_{B}=(\varepsilon-3) \phi_{A}, \\
\frac{3}{2}\left[q_{x}+p c\left(y+\frac{s}{r c}\right)-r c\left(y \partial_{y}+\frac{1}{2}\right)\right] \phi_{A}=(\varepsilon+3) \phi_{B},
\end{gathered}
$$

where $\varepsilon=\frac{E}{(J S)}$.

Eliminating $\phi_{A}$ by substituting Eq. (B7) into Eq. (B8), we obtain a second-order ordinary differential equation with variable coefficient,

$$
y^{2} \phi_{B}^{\prime \prime}+2 y \phi_{B}^{\prime}-\left[\frac{p^{2}}{r^{2}} y^{2}+\eta y+\frac{\Delta}{c^{2} r^{4}}-\frac{1}{4}\right] \phi_{B}=0,
$$

where $\Delta=\left(q_{x} r+p s\right)^{2}-4 r^{2}+r^{2}\left(\frac{2}{3} \varepsilon\right)^{2}$ and $\eta=\frac{p}{c r^{3}}\left(2 q_{x} r+\right.$ $\left.2 p s-c r^{2}\right)$. We first examine the asymptotic form of the solution. As $y \rightarrow-\infty$, the $y^{2}$ term dominates, so $\phi_{B}^{\prime \prime}-\frac{p^{2}}{r^{2}} \phi_{B}=$ 0 . The general solution is $\phi_{B}=A e^{-\frac{p}{r} y}+B e^{\frac{p}{r} y}$. Since $e^{-\frac{p}{r} y}$ diverges at $y \rightarrow-\infty, \phi_{B} \sim e^{\frac{p}{r} y}$. Similarly, at $y=0, \phi_{B} \sim$ $e^{-\frac{1}{2}+\frac{\sqrt{\Delta}}{c r^{2}}}$. Taking these asymptotic behaviors into considera-

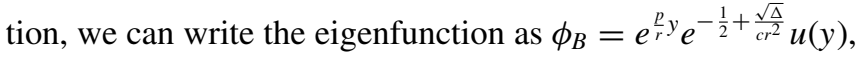
so that the differential equation can be simplified. In terms of $u(y)$, Eq. (B9) becomes

$$
\begin{aligned}
y u^{\prime \prime} & +\left(1+\frac{2 \sqrt{\Delta}}{c r^{2}}+\frac{2 p}{r} y\right) u^{\prime} \\
& +\frac{2 p}{r}\left(1+\frac{\sqrt{\Delta}}{c r^{2}}-\frac{q_{x} r+p s}{c r^{2}}\right) u=0 .
\end{aligned}
$$

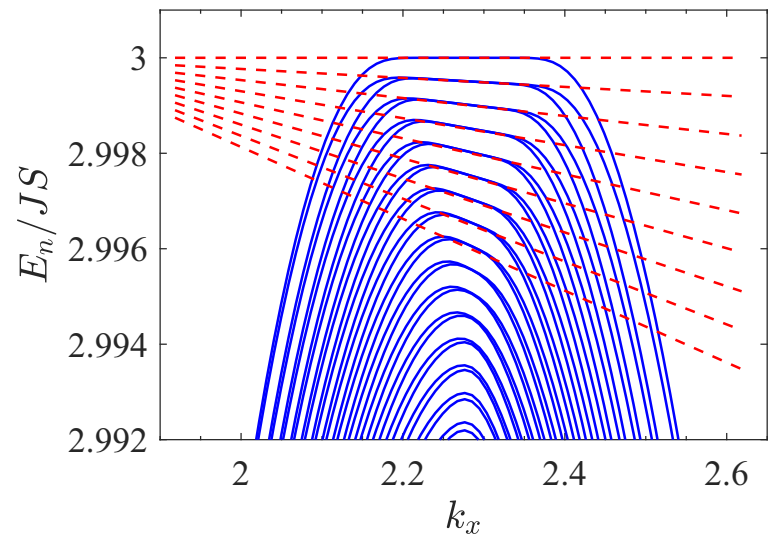

FIG. 8. Comparison between the analytical solution (B12) and the dispersion obtained by numerically diagonalizing the Hamiltonian matrix near one of the Dirac points. Here the $y$-dependent term before $\sigma_{0}$ in Eq. (B4) is dropped artificially. The strain strength is $c / c_{\max }=0.5$. The linear size used in the numerical diagonalization is $L_{y}=200$.

Introducing $\gamma=1+\frac{\sqrt{\Delta}}{c r^{2}}, \alpha=1+\frac{\sqrt{\Delta}}{c r^{2}}-\frac{q_{x} r+p s}{c r^{2}}$, and $z=$ $-\frac{2 p}{r} y$, we arrive at the confluent hypergeometric equation, $z u^{\prime \prime}(z)+(\gamma-z) u^{\prime}(z)-\alpha u(z)=0$. The above differential equation has a regular singularity at $z=0$ and can be solved by the series expansion method. One solution is

$u(z)=1+\frac{\alpha}{\gamma} \frac{z}{1 !}+\frac{\alpha(\alpha+1)}{\gamma(\gamma+1)} \frac{z^{2}}{2 !}+\cdots, \gamma \neq 0,-1,-2, \ldots$

To make $u(z)$ a polynomial so that it is finite, $\alpha$ should be 0 or a negative integer, i.e., $\alpha=-n, n=0,1,2, \ldots$. Then we get the following expression for the eigenenergy:

$$
E_{n}=3 J S \sqrt{1-\frac{2+3 q_{x}}{8} n c} .
$$

In the limit of small $c$, we can approximate the eigenenergy as $E_{n} \approx 3 J S\left(1-\frac{2+3 q_{x}}{16} n c\right)$, which implies the pseudo-Landau levels are equally spaced with the level index $n$. We compare the analytical solution with the dispersion obtained by numerically diagonalizing the Hamiltonian matrix. As shown in Fig. 8, the results are in very good consistency near the Dirac point, which further verifies our calculations in the paper.

\section{The differential equation of the full effective Hamiltonian}

Expanding the eigenvalue problem of the full effective Hamiltonian, we obtain two first-order differential equations:

$$
\begin{aligned}
& -\frac{3}{2}\left[q_{x}+p c\left(y+\frac{s}{r c}\right)+r c\left(y \partial_{y}+\frac{1}{2}\right)\right] \phi_{B}=\left\{\varepsilon-\left[3-\frac{3}{2} c\left(y+\frac{s}{r c}\right)\right]\right\} \phi_{A}, \\
& \frac{3}{2}\left[q_{x}+p c\left(y+\frac{s}{r c}\right)-r c\left(y \partial_{y}+\frac{1}{2}\right)\right] \phi_{A}=\left\{\varepsilon+\left[3-\frac{3}{2} c\left(y+\frac{s}{r c}\right)\right]\right\} \phi_{B} .
\end{aligned}
$$


Defining $\alpha(y)=\frac{3}{2} c\left(y+\frac{s}{r c}\right)$ and eliminating $\phi_{A}$ in Eq. (B14), we get the following second-order differential equation of $\phi_{B}$ :

$$
\begin{aligned}
& \left\{\left[y^{2}+\frac{\alpha}{(\varepsilon-3)} y^{2}\right] \phi_{B}^{\prime \prime}+\left[2 y+\frac{2 \alpha y-y^{2} \alpha^{\prime}(y)}{(\varepsilon-3)}\right] \phi_{B}^{\prime}\right\}-\phi_{B}\left\{\frac{y^{2}}{r^{2}}\left[p^{2}+\frac{p\left(p \alpha+r \alpha^{\prime}\right)}{(\varepsilon-3)}\right]\right. \\
& \left.+y\left[\frac{p\left(2 p s+2 q_{x} r-c r^{2}\right)}{c r^{3}}+\alpha \frac{p\left(2 p s+2 q_{x} r-c r^{2}\right)}{c r^{3}(\varepsilon-3)}+\alpha^{\prime} \frac{\left(c r^{2}+2 p s+2 q_{x} r\right)}{2 c r^{2}(\varepsilon-3)}\right]\right\} \\
& -\phi_{B}\left\{\frac{\left(p s+q_{x} r\right)^{2}}{c^{2} r^{4}}-\frac{1}{4}+\alpha \frac{4\left(p s+q_{x} r\right)^{2}-c^{2} r^{4}}{4 c^{2} r^{4}(\varepsilon-3)}\right\} \\
& \quad=-\frac{4}{9 c^{2} r^{2}}\left\{\left(\varepsilon^{2}-9\right)+\frac{[(\varepsilon+3)-\alpha]}{(\varepsilon-3)}\left[2 \alpha(\varepsilon-3)+\alpha^{2}\right]-\alpha(\varepsilon-3)\right\} \phi_{B} .
\end{aligned}
$$

If $\alpha=0$ and $\alpha^{\prime}=0$ is set, the above equation reduces to Eq. (B9). Due to the presence of the $y$-dependent term before $\sigma_{0}$, the differential equation becomes much more complex. Defining $b=\frac{3}{2} c /\left(\varepsilon-3+\frac{3 s}{2 r}\right)$, Eq. (B15) becomes

$$
\left(y^{2}+b y^{3}\right) \phi_{B}^{\prime \prime}+\left(2 y+b y^{2}\right) \phi_{B}^{\prime}-A_{0} \phi_{B},
$$

with

$$
\begin{aligned}
A_{0}= & b \frac{\left(p^{2}-1\right)}{r^{2}} y^{3}+\left\{\frac{2 b\left[p\left(p s+q_{x} r\right)-(s-2 r)\right]}{c r^{3}}+\frac{p^{2}-1}{r^{2}}\right\} y^{2} \\
& +\left(\frac{4 b \varepsilon^{2}}{9 c^{2} r^{2}}+\frac{b\left(p s+q_{x} r\right)^{2}-b(s-2 r)^{2}}{c^{2} r^{4}}+\frac{b\left(p s+q_{x} r\right)}{c r^{2}}+\frac{2\left[p\left(p s+q_{x} r\right)-(s-2 r)\right]}{c r^{3}}+\frac{b}{4}-\frac{p}{r}\right) y \\
& +\frac{4 \varepsilon^{2}}{9 c^{2} r^{2}}+\frac{\left(p s+q_{x} r\right)^{2}-(s-2 r)^{2}}{c^{2} r^{4}}-\frac{1}{4} .
\end{aligned}
$$

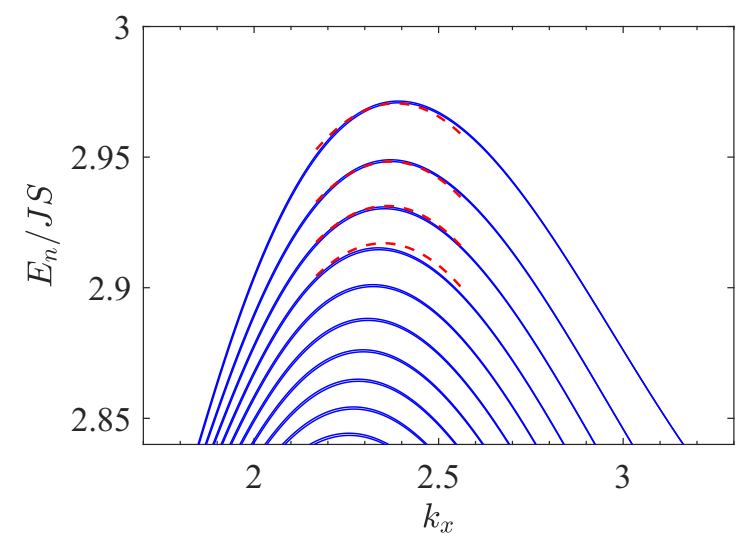

FIG. 9. Fitting the dispersion in Fig. 2(b) near the Dirac point with the formula in Eq. (B17). The fitting parameters are $a=$ $0.379, b=0.0259, c=0.0549$.
We have tried to decouple the solution using the asymptotic forms at $y=0$ and $y \rightarrow-\infty$. However, the resulting differential equation does not fit into any standard one. At present, it is still unclear whether the differential equation (B17) has an analytical solution.

\section{Fitting the dispersion in Fig. 2(b) with a square-root relation}

To further verify the square-root relation of PLLs in the strained Heisenberg Hamiltonian, we fit the dispersion in Fig. 2(b) near the Dirac point with the following formula:

$$
E_{n} / J S=3.025-a q_{x}^{2}-\sqrt{n}\left(b q_{x}+c\right) .
$$

As shown in Fig. 9, the square-root relation offers a pretty good fit of several leading PLLs.
[1] V. M. Pereira and A. H. Castro Neto, Strain Engineering of Graphene's Electronic Structure, Phys. Rev. Lett. 103, 046801 (2009).

[2] F. Guinea, M. Katsnelson, and A. Geim, Energy gaps and a zero-field quantum Hall effect in graphene by strain engineering, Nat. Phys. 6, 30 (2010).

[3] L. Yang, X. Jiang, Z. Liu, and Z. Liu, Strain effects in graphene and graphene nanoribbons: The underlying mechanism, Nano Res. 3, 581 (2010).

[4] B. Amorim, A. Cortijo, F. de Juan, A. Grushin, F. Guinea, A. Gutiérrez-Rubio, H. Ochoa, V. Parente, R. Roldán, P. San-Jose et al., Novel effects of strains in graphene and other two dimensional materials, Phys. Rep. 617, 1 (2016).
[5] F. de Juan, J. L. Mañes, and M. A. H. Vozmediano, Gauge fields from strain in graphene, Phys. Rev. B 87, 165131 (2013).

[6] A. H. Castro Neto, F. Guinea, N. M. R. Peres, K. S. Novoselov, and A. K. Geim, The electronic properties of graphene, Rev. Mod. Phys. 81, 109 (2009).

[7] V. M. Pereira, A. H. Castro Neto, and N. M. R. Peres, Tightbinding approach to uniaxial strain in graphene, Phys. Rev. B 80, 045401 (2009).

[8] T. M. G. Mohiuddin, A. Lombardo, R. R. Nair, A. Bonetti, G. Savini, R. Jalil, N. Bonini, D. M. Basko, C. Galiotis, N. Marzari et al., Uniaxial strain in graphene by Raman spectroscopy: $G$ peak splitting, Grüneisen parameters, and sample orientation, Phys. Rev. B 79, 205433 (2009). 
[9] M. Neek-Amal, L. Covaci, K. Shakouri, and F. M. Peeters, Electronic structure of a hexagonal graphene flake subjected to triaxial stress, Phys. Rev. B 88, 115428 (2013).

[10] M. Settnes, S. R. Power, and A.-P. Jauho, Pseudomagnetic fields and triaxial strain in graphene, Phys. Rev. B 93, 035456 (2016).

[11] F. Guinea, A. K. Geim, M. I. Katsnelson, and K. S. Novoselov, Generating quantizing pseudomagnetic fields by bending graphene ribbons, Phys. Rev. B 81, 035408 (2010).

[12] Y. Chang, T. Albash, and S. Haas, Quantum Hall states in graphene from strain-induced nonuniform magnetic fields, Phys. Rev. B 86, 125402 (2012).

[13] D.-B. Zhang, G. Seifert, and K. Chang, Strain-Induced Pseudomagnetic Fields in Twisted Graphene Nanoribbons, Phys. Rev. Lett. 112, 096805 (2014).

[14] Y.-H. Ho, E. V. Castro, and M. A. Cazalilla, Haldane model under nonuniform strain, Phys. Rev. B 96, 155446 (2017).

[15] N. Levy, S. A. Burke, K. L. Meaker, M. Panlasigui, A. Zettl, F. Guinea, A. H. Castro Neto, and M. F. Crommie, Strain-induced pseudo-magnetic fields greater than 300 Tesla in graphene nanobubbles, Science 329, 544 (2010).

[16] J. Mao, S. P. Milovanović, M. Andelković, X. Lai, Y. Cao, K. Watanabe, T. Taniguchi, L. Covaci, F. M. Peeters, A. K. Geim et al., Evidence of flat bands and correlated states in buckled graphene superlattices, Nature (London) 584, 215 (2020).

[17] L. Meng, W.-Y. He, H. Zheng, M. Liu, H. Yan, W. Yan, Z.-D. Chu, K. Bai, R.-F. Dou, Y. Zhang et al., Strain-induced onedimensional Landau level quantization in corrugated graphene, Phys. Rev. B 87, 205405 (2013).

[18] D. I. Pikulin, A. Chen, and M. Franz, Chiral Anomaly from Strain-Induced Gauge Fields in Dirac and Weyl Semimetals, Phys. Rev. X 6, 041021 (2016).

[19] T. Liu, M. Franz, and S. Fujimoto, Quantum oscillations and Dirac-Landau levels in Weyl superconductors, Phys. Rev. B 96, 224518 (2017).

[20] T. Liu, D. I. Pikulin, and M. Franz, Quantum oscillations without magnetic field, Phys. Rev. B 95, 041201 (2017).

[21] E. M. Nica and M. Franz, Landau levels from neutral Bogoliubov particles in two-dimensional nodal superconductors under strain and doping gradients, Phys. Rev. B 97, 024520(R) (2018).

[22] B. Li and A. A. Kovalev, Magnon Landau Levels and Spin Responses in Antiferromagnets, Phys. Rev. Lett. 125, 257201 (2020).

[23] T. Liu and Z. Shi, Strain-induced dispersive Landau levels: Application in twisted honeycomb magnets, Phys. Rev. B 103, 144420 (2021).

[24] S. Rachel, L. Fritz, and M. Vojta, Landau Levels of Majorana Fermions in a Spin Liquid, Phys. Rev. Lett. 116, 167201 (2016).

[25] J. Guglielmon, M. C. Rechtsman, and M. I. Weinstein, Landau levels in strained two-dimensional photonic crystals, Phys. Rev. A 103, 013505 (2021).

[26] Z. Yang, F. Gao, Y. Yang, and B. Zhang, Strain-Induced Gauge Field and Landau Levels in Acoustic Structures, Phys. Rev. Lett. 118, 194301 (2017).

[27] M. M. Nayga, S. Rachel, and M. Vojta, Magnon Landau Levels and Emergent Supersymmetry in Strained Antiferromagnets, Phys. Rev. Lett. 123, 207204 (2019).

[28] J. Sun, N. Ma, T. Ying, H. Guo, and S. Feng, Quantum Monte Carlo study of honeycomb antiferromagnets under a triaxial strain, Phys. Rev. B 104, 125117 (2021).
[29] E. Lantagne-Hurtubise, X.-X. Zhang, and M. Franz, Dispersive Landau levels and valley currents in strained graphene nanoribbons, Phys. Rev. B 101, 085423 (2020).

[30] O. F. Syljuåsen and A. W. Sandvik, Quantum Monte Carlo with directed loops, Phys. Rev. E 66, 046701 (2002).

[31] O. F. Syljuåsen, Directed loop updates for quantum lattice models, Phys. Rev. E 67, 046701 (2003).

[32] B. Bauer, L. D. Carr, H. G. Evertz, A. Feiguin, J. Freire, S. Fuchs, L. Gamper, J. Gukelberger, E. Gull, S. Guertler et al., The ALPS project release 2.0: open source software for strongly correlated systems, J. Stat. Mech.: Theory Exp. (2011) P05001.

[33] F. Alet, S. Wessel, and M. Troyer, Generalized directed loop method for quantum Monte Carlo simulations, Phys. Rev. E 71, 036706 (2005).

[34] L. Pollet, S. M. A. Rombouts, K. Van Houcke, and K. Heyde, Optimal Monte Carlo updating, Phys. Rev. E 70, 056705 (2004).

[35] T. Holstein and H. Primakoff, Field dependence of the intrinsic domain magnetization of a ferromagnet, Phys. Rev. 58, 1098 (1940).

[36] R. M. White, M. Sparks, and I. Ortenburger, Diagonalization of the antiferromagnetic magnon-phonon interaction, Phys. Rev. 139, A450 (1965).

[37] M.-W. Xiao, Theory of transformation for the diagonalization of quadratic Hamiltonians, arXiv:0908.0787.

[38] W. M. Huang, T. Hikihara, Y. C. Lee, and H. H. Lin, Edge magnetism of Heisenberg model on honeycomb lattice, Sci. Rep. 7, 43678 (2017).

[39] G. Sala, M. B. Stone, B. K. Rai, A. F. May, P. Laurell, V. O. Garlea, N. P. Butch, M. Lumsden, G. Ehlers, and G. Pokharel, Van Hove singularity in the magnon spectrum of the antiferromagnetic quantum honeycomb lattice, Nat. Commun. 12, 171 (2021).

[40] M. O. Goerbig, Electronic properties of graphene in a strong magnetic field, Rev. Mod. Phys. 83, 1193 (2011).

[41] S. Wessel and I. Milat, Quantum fluctuations and excitations in antiferromagnetic quasicrystals, Phys. Rev. B 71, 104427 (2005).

[42] T. Hikihara, X. Hu, H.-H. Lin, and C.-Y. Mou, Ground-state properties of nanographite systems with zigzag edges, Phys. Rev. B 68, 035432 (2003).

[43] M. Golor, S. Wessel, and M. J. Schmidt, Quantum Nature of Edge Magnetism in Graphene, Phys. Rev. Lett. 112, 046601 (2014).

[44] M. Golor, T. C. Lang, and S. Wessel, Quantum Monte Carlo studies of edge magnetism in chiral graphene nanoribbons, Phys. Rev. B 87, 155441 (2013).

[45] H. Feldner, Z. Y. Meng, A. Honecker, D. Cabra, S. Wessel, and F. F. Assaad, Magnetism of finite graphene samples: Mean-field theory compared with exact diagonalization and quantum Monte Carlo simulations, Phys. Rev. B 81, 115416 (2010).

[46] B. Roy, F. F. Assaad, and I. F. Herbut, Zero Modes and Global Antiferromagnetism in Strained Graphene, Phys. Rev. X 4, 021042 (2014).

[47] S. Wessel, A. Jagannathan, and S. Haas, Quantum Antiferromagnetism in Quasicrystals, Phys. Rev. Lett. 90, 177205 (2003).

[48] B. Dalla Piazza, M. Mourigal, N. B. Christensen, G. Nilsen, P. Tregenna-Piggott, T. Perring, M. Enderle, D. F. McMorrow, 
D. Ivanov, and H. M. Rønnow, Fractional excitations in the square-lattice quantum antiferromagnet, Nat. Phys. 11, 62 (2015).

[49] H. Shao, Y. Q. Qin, S. Capponi, S. Chesi, Z. Y. Meng, and A. W. Sandvik, Nearly Deconfined Spinon Excitations in the SquareLattice Spin-1/2 Heisenberg Antiferromagnet, Phys. Rev. X 7, 041072 (2017).

[50] G. Gomez-Santos and J. D. Joannopoulos, Application of spinwave theory to the ground state of XY quantum Hamiltonians, Phys. Rev. B 36, 8707 (1987).

[51] J. Guo, J. Sun, X. Zhu, C.-A. Li, H. Guo, and S. Feng, Quantum Monte Carlo study of topological phases on a spin analogue of Benalcazar-Bernevig-Hughes model, J. Phys.: Condens. Matter 34, 035603 (2021).

[52] Y.-L. Zhang, Y.-Y. Zhang, J.-Y. Ni, J.-H. Yang, H.-J. Xiang, and X.-G. Gong, Rational design of two-dimensional magnetic chromium borides based on first-principles calculation, Chin. Phys. Lett. 38, 027501 (2021).

[53] K. S. Burch, D. Mandrus, and J.-G. Park, Magnetism in twodimensional van der Waals materials, Nature (London) 563, 47 (2018).

[54] M. Gibertini, M. Koperski, A. Morpurgo, and K. Novoselov, Magnetic 2D materials and heterostructures, Nat. Nanotechnol. 14, 408 (2019).

[55] B. Yuan, I. Khait, G.-J. Shu, F. C. Chou, M. B. Stone, J. P. Clancy, A. Paramekanti, and Y.-J. Kim, Dirac Magnons in a Honeycomb Lattice Quantum $X Y$ Magnet $\mathrm{CoTiO}_{3}$, Phys. Rev. X 10, 011062 (2020).

[56] J.-U. Lee, S. Lee, J. H. Ryoo, S. Kang, T. Y. Kim, P. Kim, C.-H. Park, J.-G. Park, and H. Cheong, Ising-type magnetic ordering in atomically thin $\mathrm{FePS}_{3}$, Nano Lett. 16, 7433 (2016).

[57] X. Wang, K. Du, Y. Y. F. Liu, P. Hu, J. Zhang, Q. Zhang, M. H. S. Owen, X. Lu, C. K. Gan, P. Sengupta et al., Raman spectroscopy of atomically thin two-dimensional magnetic iron phosphorus trisulfide $\left(\mathrm{FePS}_{3}\right)$ crystals, 2D Mater. 3, 031009 (2016).
[58] C. Gong, L. Li, Z. Li, H. Ji, A. Stern, Y. Xia, T. Cao, W. Bao, C. Wang, Y. Wang et al., Discovery of intrinsic ferromagnetism in two-dimensional van der Waals crystals, Nature (London) 546, 265 (2017).

[59] M. Bonilla, S. Kolekar, Y. Ma, H. C. Diaz, V. Kalappattil, R. Das, T. Eggers, H. R. Gutierrez, M.-H. Phan, and M. Batzill, Strong room-temperature ferromagnetism in $\mathrm{VSe}_{2}$ monolayers on van der Waals substrates, Nat. Nanotechnol. 13, 289 (2018).

[60] D. J. O’Hara, T. Zhu, A. H. Trout, A. S. Ahmed, Y. K. Luo, C. H. Lee, M. R. Brenner, S. Rajan, J. A. Gupta, D. W. McComb et al., Room temperature intrinsic ferromagnetism in epitaxial manganese selenide films in the monolayer limit, Nano Lett. 18 3125 (2018).

[61] S. I. Vishkayi, Z. Torbatian, A. Qaiumzadeh, and R. Asgari, Strain and electric-field control of spin-spin interactions in monolayer $\mathrm{CrI}_{3}$, Phys. Rev. Mater. 4, 094004 (2020).

[62] T. Mukherjee, S. Chowdhury, D. Jana, and L. C. L. Y. Voon, Strain induced electronic and magnetic properties of 2D magnet $\mathrm{CrI}_{3}$ : a DFT approach, J. Phys.: Condens. Matter 31, 335802 (2019).

[63] Y. Wang, C. Wang, S.-J. Liang, Z. Ma, K. Xu, X. Liu, L. Zhang, A. S. Admasu, S.-W. Cheong, L. Wang et al., Strain-sensitive magnetization reversal of a van der Waals magnet, Adv. Mater. 32, 2004533 (2020).

[64] R. Roldán, A. Castellanos-Gomez, E. Cappelluti, and F. Guinea, Strain engineering in semiconducting two-dimensional crystals, J. Phys.: Condens. Matter 27, 313201 (2015).

[65] A. V. Chumak, V. I. Vasyuchka, A. A. Serga, and B. Hillebrands, Magnon spintronics, Nat. Phys. 11, 453 (2015).

[66] W. Xing, L. Qiu, X. Wang, Y. Yao, Y. Ma, R. Cai, S. Jia, X. C. Xie, and W. Han, Magnon Transport in Quasi-TwoDimensional van der Waals Antiferromagnets, Phys. Rev. X 9, 011026 (2019). 